\title{
Overview and performance prediction of the baseline 4-meter telescope concept design for the habitable-zone exoplanet observatory

\author{
H. Philip Stahl,
}

${ }^{a}$ NASA MARSHALL SPACE FLIGTH CENTER, HUNTSVILLE, AL 35812

\begin{abstract}
The Habitable Exoplanet Observatory Mission (HabEx) is one of four missions under study for the 2020 Astrophysics Decadal Survey. Its goal is to directly image and spectroscopically characterize planetary systems in the habitable zone around nearby sun-like stars. Additionally, HabEx will perform a broad range of general astrophysics science enabled by 100 to $2500 \mathrm{~nm}$ spectral range and $3 \times 3$ arc-minute FOV. Critical to achieving the HabEx science goals is a large, ultrastable UV/Optical/Near-IR (UVOIR) telescope. The baseline HabEx telescope is a 4-meter off-axis unobscured threemirror-anastigmatic, diffraction limited at $400 \mathrm{~nm}$ with wavefront stability on the order of a few $10 \mathrm{~s}$ of picometers. This paper summarizes the opto-mechanical design of the HabEx baseline optical telescope assembly, including a discussion of how science requirements drive the telescope's specifications, and presents analysis that the baseline telescope structure meets its specified tolerances.
\end{abstract}

Keywords: space telescopes, astrophysics, astronomy, HabEx

\section{INTRODUCTION}

"Are we alone in the Universe?" is probably the most compelling science question of our generation. Per the 2010 New Worlds, New Horizons Decadal Report ${ }^{1}$ : "One of the fastest growing and most exciting fields in astrophysics is the study of planets beyond our solar system. The ultimate goal is to image rocky planets that lie in the habitable zone of nearby stars." The Survey recommended, as its highest priority, medium-scale activity such as a "New Worlds Technology Development Program" to "lay the technical and scientific foundations for a future space imaging and spectroscopy mission." The National Research Council report, NASA Space Technology Roadmaps \& Prioroties $^{2}$, states that the second highest technical challenge for NASA regarding expanding our understanding of Earth and the universe in which we live is to "Develop a new generation of astronomical telescopes that enable discovery of habitable planets, facilitate advances in solar physics, and enable the study of faint structures around bright objects by developing high-contrast imaging and spectroscopic technologies to provide unprecedented sensitivity, field of view, and spectroscopy of faint objects."

As a result, NASA is studying in detail the Habitable Exoplanet Observatory Mission (HabEx) for the 2020 Decadal Survey. ${ }^{3,4}$ HabEx has three goals: to seek out nearby worlds and explore their habitability; to map out nearby planetary systems and understand the diversity of the worlds they contain; and, to carry out observations that open up new windows on the universe from the UV through near-IR. The HabEx Science and Technology Definition Team has selected as 'Architecture A' a 4-meter telescope with four science instruments (coronagraph, star-shade instrument, UV-NIR imaging multi-object slit spectrograph, and a high resolution UV spectrograph; and a 72-m external star-shade occulter.

Telescope design is an iterative process. In 2017, we published a paper that provided an overview of an initial HabEx telescope design concept, our design process and a performance evaluation for the concept. ${ }^{5}$ Since that paper, the telescope design was modified extensively to become the 'baseline' design. And currently, the baseline design is being refined to become the 'Rev 1' design. This paper reviews the design process for the baseline design, summarizes its design features and presents performance prediction analysis. Section 2 reviews how the HabEx OTA specifications are derived from the HabEx science requirements. And, how the HabEx OTA specifications are primarily driven by requirements imposed by the coronagraph. Section 3 describes how the system level specifications are flown into opto-mechanical tolerances for rigid body motions. Section 4 provides an overview of the baseline opto-mechanical OTA design. Finally, Section 5 summarizes detailed dynamic analysis of the baseline opto-mechanical design which shows that the design, using proven technology and engineering practicw, can achieve the performance specifications necessary to perform HabEx science. The baseline 4-m off-axis HabEx opto-mechanical telescope design 'closes' for its line-of-sight (LOS) and wavefront error (WFE) stability specifications. The only external assumptions is that the mission is launched in an SLS 8.4-m fairing and uses low disturbance micro-thrusters for pointing control.

Stahl, H. Philip, "Overview and performance prediction of the baseline 4-meter telescope concept design for the habitable-zone exoplanet observatory”, Space Telescopes and Instrumentation 2018, SPIE 10698-31, 2018. 


\section{OPTICAL TELESCOPE ASSEMBLY SPECIFICATIONS}

The HabEx Optical Telescope Assembly (OTA) design (optical and structural) specifications are mostly driven by the needs of exoplanet science using an internal coronagraph. To image exoplanets in the habitable zone close to their host star using a coronagraph requires a telescope/coronagraph 'system' that can produce a $10^{-10}$ 'dark hole' with as small of an inner working angle (IWA) as possible and as large of an irradiance throughput as possible. The smaller the IWA and the larger the throughput, the greater the number of habitable zones that can be searched - the greater the science 'yield'. IWA is the minimum angular distance (on the sky) where the 'dark hole' begins - the location when the coronagraph can block $10^{10}$ of the host stars light (Figure 1). The ability to achieve a small IWA depends upon the telescope's ability to produce a small stable point spread function (PSF) with a compact stable encircled energy (EE). The smaller the EE, the smaller the IWA. The remaining specifications

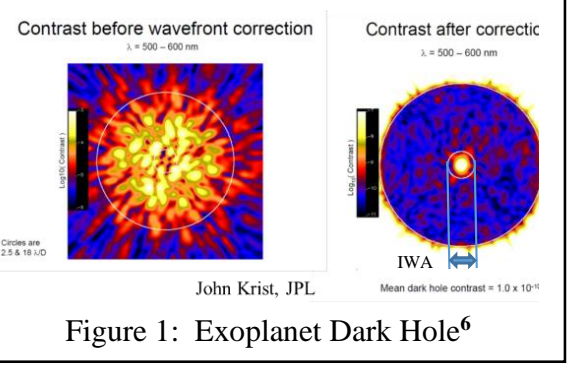
are provided by the desire to perform wide-field general astrophysics. Table 1 summarizes the HabEx OTA specifications.

\begin{tabular}{|c|c|}
\hline \multicolumn{2}{|c|}{ Table 1: HabEx Optical Telescope Specification } \\
\hline Specification & Value \\
\hline Architecture & Off-Axis Unobscured Circular Aperture \\
\hline Optical Design & Three-Mirror Anastigmatic \\
\hline Science Instruments & On the side, in the Secondary Mirror Tower structure \\
\hline Aperture Diameter & $>4.0$ meters \\
\hline Primary Mirror F/\# & F/2.5 or slower \\
\hline Diffraction Limited Wavelength & $400 \mathrm{~nm}$ \\
\hline Observatory WFE & $<35 \mathrm{~nm} \mathrm{rms}$ \\
\hline OTA WFE & $<30 \mathrm{~nm} \mathrm{rms}$ \\
\hline PMA SFE & $<8 \mathrm{~nm} \mathrm{rms}$ \\
\hline Low Spatial SFE (<3 cycles/diameter) & $<5.6 \mathrm{~nm} \mathrm{rms}$ \\
\hline Mid Spatial SFE (3 to 60 cycles/diameter) & $<5.6 \mathrm{~nm} \mathrm{rms}$ \\
\hline High Spatial SFE (> 60 cycles/diameter) & $<0.6 \mathrm{~nm} \mathrm{rms}$ \\
\hline Roughness & $<0.2 \mathrm{~nm} \mathrm{rms}$ \\
\hline Wavefront Error Stability & 1 to $250 \mathrm{pm}$ depending on coronagraph and spatial frequency \\
\hline Line of Sight Stability (Jitter) & $<0.5$ milli-arc-seconds per axis \\
\hline
\end{tabular}

The IWA requirement drives two system specifications: aperture diameter and off-axis configuration. It is common knowledge that the larger a telescope's aperture, the smaller its point spread function (PSF) and its Encircled Energy (EE). But, what is often overlooked is that an unobscured (off-axis) telescope always has a more compact EE (better IWA) than an on-axis telescope with a central obscuration - because diffraction from the central obscuration broadens the PSF. To be specific, an unobscured circular aperture has $82.8 \% \mathrm{EE}$ at $\lambda / \mathrm{D}$; for a $10 \%$ central obscuration, $82.5 \% \mathrm{EE}$ is at $1.4 \lambda / \mathrm{D}$; and, for a $20 \%$ obscuration, $82 \% \mathrm{EE}$ is at $1.63 \lambda / \mathrm{D}^{7}$ Thus to achieve similar IWA performance as an unobscured 4-m telescope, an on-axis telescope with $10 \%$ central obscuration would need to be at least $5.6-\mathrm{m}$ and one with $20 \%$ obscuration would need to be at least $6.5-\mathrm{m}$. Additionally, diffraction from secondary mirror spider obscurations also distort the PSF and broaden the EE. A 1 to $2 \%$ wide spider can increase EE diameter (IWA) by 5 to $10 \%{ }^{7}$ - requiring a 5 to $10 \%$ larger on-axis telescope. Of course the problem is even worse for a segmented aperture primary mirror. Thus, the baseline HabEx optical telescope is an unobscured off-axis monolithic aperture configuration.

Because general astrophysics science desires a $3 \times 3$ arcminute field of view (FOV) for its imager and multi-object spectrograph, the HabEx is baselining a three mirror anastigmatic (TMA) design.

Regarding minimum aperture, based on a design reference mission yield estimate for an off-axis-telescope/coronagraph combination, the minimum desired aperture diameter is 4 -meter. ${ }^{8}$ And, while a larger aperture would provide higher yield, 4-m was selected as the baseline for several programmatic reasons. First, 4-m class mirrors are manufacturable. Schott has an existing infrastructure to melt and cast $4.2-\mathrm{m}$ diameter by $42 \mathrm{~cm}$ thick Zerodur® mirror substrates. And, Corning has the infrastructure to either frit bond or low-temperature-fuse 4-m 'class' ULE® mirror substrates. And at least four organizations have existing infrastructure to grind and polish 4-m class substrates into space mirrors, including: United Technology Optical Systems in Danbury CT, L3/Brashears in Pittsburgh, University of Arizona in Tucson and RESOC outside of Paris France. Second, a 4-m class telescope can be packaged inside of NASA's planned SLS 8.4-m fairing. 
Potentially the most important specification driving the telescope design is the primary mirror's F/\#. From a packaging perspective, a fast PM F/\# or short radius of curvature is desired. But, to minimize polarization cross-talk in the coronagraph, a slow PM F/\# is desired. After consideration, an optical design similar to Exo-C with an $\mathrm{F} / 2.5$ primary mirror and the science instruments located on the anti-Sun side of the telescope ${ }^{9}$ was selected. This configuration minimizes the need for high incidence angle reflections that produce unwanted polarization effects and isolates the coronagraph from thermal disturbances. As a consequence, the HabEx OTA is physically long. The baseline 4-m design has a primary to secondary mirror separation of $\sim 9-\mathrm{m}$.

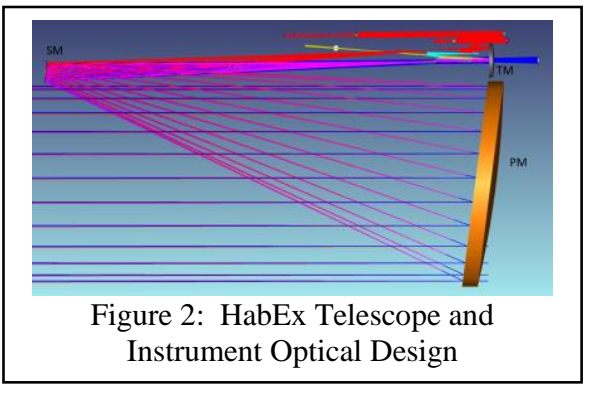

Finally, the optical telescope wavefront error specification and primary mirror flow-down allocation are derived using standard methods. The specification is based on a desired $400 \mathrm{~nm}$ diffraction limited performance. The primary mirror allocation assumes computer controlled polishing for low spatial frequencies and a -2 slope for high spatial frequencies.

\subsection{Line of Sight (LOS) Stability Specification}

LOS jitter is important for both general astrophysics and coronagraphy because it causes PSF smearing that degrades spatial resolution and IWA. A typical specification for LOS jitter is less than 1/10th the point spread function (PSF) radius. For a $400 \mathrm{~nm}$ diffraction limited 4-m telescope, the on-sky PSF radius is 25 mas. Thus, the jitter specification should be $<2.5$ mas. But, coronagraphs require better LOS stability. The reason is that jitter causes beam-shear on the secondary and tertiary mirrors, as well as other mirrors in the optical train, which introduces WFE that result in contrast leakage. Assuming that the telescope may have a laser-truss system and/or a low-order wavefront-sensor (LOWFS) that can sense and correct LOS drift/vibrations with amplitudes on the order of 2.5 mas to less than 0.5 mas at frequencies below $10 \mathrm{~Hz}$, the LOS stability specification ${ }^{\mathbf{1 0}}$ has temporal regimes (Table 2 )

\begin{tabular}{|l|l|l|l|}
\hline \multicolumn{2}{|c|}{ Table 2: HabEx Line of Sight Specification } \\
\hline Temporal Frequency & Jitter Amplitude per Axis \\
\hline$<10 \mathrm{~Hz}$ & & $<1$ milli-arc-seconds \\
\hline$>10 \mathrm{~Hz}$ & & $<0.5$ milli-arc-seconds (only required for Coronagraphy) \\
\hline
\end{tabular}

Please note that these LOS stability specifications depend on the exact optical prescriptions of all mirrors in the optical train and their fabrication quality, i.e. residual low-order and mid-spatial frequency errors.

To be conservative, for the purpose of designing the HabEx telescope opto-mechanical structure, the telescope design team is assuming that the telescope must have a LOS stability that meets the ' $>10 \mathrm{~Hz}$ ' requirement. Current design analysis indicates that all HabEx rigid body modes affecting LOS jitter occur at frequencies above $20 \mathrm{~Hz}$. Thus, all telescope jitter is uncorrectable by either the spacecraft's attitude control system (which on WFIRST has a bandwidth of $0.05 \mathrm{~Hz}$ ) or a low-order wavefront sensor (WFIRST's LOWFS has a bandwidth of approximately $10 \mathrm{~Hz}$ ). ${ }^{\mathbf{1 0}}$ Thus, the 'on-sky' LOS Stability specification for the HabEx optical telescope assembly is < 0.5 mas per axis. And, for the current HabEx optical design with its $80 \mathrm{X}$ magnification, the LOS Stability specification at the FSM is $<40$ mas.

\subsection{Wavefront Error (WFE) Stability Specification}

The WFE stability specification is driven by the coronagraph. Any temporal or dynamic change in WFE can result in dark-hole speckles that produce a false exoplanet measurement or mask a true signal. The issue is how large of a WFE can any given coronagraph tolerate. A leading candidate is the Vector Vortex Coronagraph (VVC-N) where $\mathrm{N}$ indicates the 'charge' or azimuthal shear. The higher the 'charge' the more low order error it can tolerate, but the larger its IWA and the lower its throughput. Currently, HabEx is baselining the VVC-6. Figure 3 summarizes the maximum amount of each Zernike polynomial that can be tolerated by VVC-4 to VVC-10. ${ }^{11}$ Please note that the tolerance depends on the WFE spatial frequency.

WFE instability arises from mechanical and thermal sources. Thermal errors occur when a telescope is slewed relative to the Sun. Thermal load changes cause the structure holding the mirrors to expand/contract

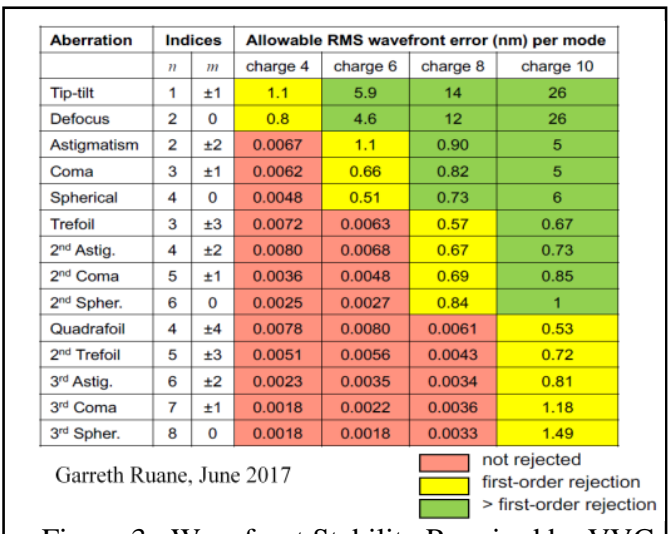

Figure 3: Wavefront Stability Required by VVC 
(resulting in alignment drift) and the mirrors themselves to change shape. Fortunately, thermal effects are slow and can be corrected. The telescope design team assumes that any coronagraph will have a wavefront sensing and control (WFSC) capability - such that a sensor will quantify any change in WFE and command deformable mirrors (DMs) in the coronagraph to correct any change - to maintain the dark hole. The problem is that WFSC is not instantaneous. There is an update period, and the telescope must be stable at the required $\mathrm{pm}$ level for the duration of the update period. Depending upon the magnitude of the host star whose planetary system is being investigated, this update period may range from 10 to 20 to 30 minutes. To mitigate this issue, the telescope design team is baselining low coefficient of thermal expansion materials such as Zerodur ${ }^{\circledR}$ ceramic or Ultra-Low Expansion (ULE®) glass. Additionally, we have baselined a predictive thermal control technology active temperature control system ${ }^{12}$ to keep the telescope at a constant temperature.

Another source of WFE instability is mechanical disturbance. Mechanical forces (from reaction wheels, cryo-coolers, etc.) can excite vibrational modes and inertial motion in the mirrors and structure that holds them. Again, temporal frequency is important. WFIRST plans a LOWFS to sense and correct low-order errors. But, its bandwidth is only about $10 \mathrm{~Hz}$. Current analysis of the baseline HabEx opto-mechanical structure indicates that all rigid body modes causing WFE stability occur at frequencies above $20 \mathrm{~Hz}$ and are thus uncorrectable. However, there is one mitigating factor. While all mechanical vibration is in general bad, there are degrees of badness. If the motions are perfectly periodic, multiple cycles over an integration period will produce a fixed pattern. If this pattern is $100 \%$ repeatable, it is possible to remove it through 'speckle subtraction'. But, if the vibration is not perfectly periodic, there will be a non-repeatable component to the error that cannot be calibrated and removed.

Given that the WFE in Figure 3 are the maximum allowed for each term, an error budget was defined for the optical telescope assembly as a function of Zernike polynomials and allocated between LOS, Inertial and Thermal (Figure 4). The allocation is accomplished in RSS space.

\begin{tabular}{|c|c|c|c|c|c|c|c|c|}
\hline & & & RSS Allocation & $100 \%$ & $50 \%$ & $60 \%$ & $60 \%$ & $20 \%$ \\
\hline \multicolumn{3}{|c|}{ Order } & & VVC-6 Allowable & LOS & Inertial & Thermal & Reserve \\
\hline \multirow[t]{2}{*}{$\mathrm{K}$} & $\mathrm{N}$ & $M$ & Aberration & [pm rms] & [pm rms] & [pm rms] & [pm rms] & [pm rms] \\
\hline & & & TOTAL RMS & 416 & 208 & 250 & 250 & 83 \\
\hline 2 & 1 & 1 & Tilt & & 0 & 0 & 0 & 0 \\
\hline 3 & 2 & 0 & Power (Defocus) & 250 & 125 & 150 & 150 & 50 \\
\hline 4 & 2 & 2 & Pri Astigmatism & 200 & 100 & 120 & 120 & 40 \\
\hline 5 & 3 & 1 & Pri Coma & 175 & 87.5 & 105 & 105 & 35 \\
\hline 6 & 4 & 0 & Pri Spherical & 200 & 100 & 120 & 120 & 40 \\
\hline 7 & 3 & 3 & Pri Trefoil & 2.6 & 1.3 & 1.56 & 1.56 & 0.52 \\
\hline 8 & 4 & 2 & Sec Astigmatism & 0.35 & 0.175 & 0.21 & 0.21 & 0.07 \\
\hline 9 & 5 & 1 & Sec Coma & 0.35 & 0.175 & 0.21 & 0.21 & 0.07 \\
\hline 10 & 6 & 0 & Sec Spherical & 0.35 & 0.175 & 0.21 & 0.21 & 0.07 \\
\hline 11 & 4 & 4 & Pri Tetrafoil & 0.35 & 0.175 & 0.21 & 0.21 & 0.07 \\
\hline 12 & 5 & 3 & Sec Trefoil & 0.35 & 0.175 & 0.21 & 0.21 & 0.07 \\
\hline 13 & 6 & 2 & Ter Astigmatism & 0.1 & 0.05 & 0.06 & 0.06 & 0.02 \\
\hline 14 & 7 & 1 & Ter Coma & 0.1 & 0.05 & 0.06 & 0.06 & 0.02 \\
\hline 15 & 8 & 0 & Ter Spherical & 0.1 & 0.05 & 0.06 & 0.06 & 0.02 \\
\hline 16 & 5 & 5 & Pri Pentafoil & 0.35 & 0.175 & 0.21 & 0.21 & 0.07 \\
\hline 17 & 6 & 4 & Sec Tetrafoil & 0.1 & 0.05 & 0.06 & 0.06 & 0.02 \\
\hline 18 & 7 & 3 & Ter Trefoil & 0.1 & 0.05 & 0.06 & 0.06 & 0.02 \\
\hline 19 & 8 & 2 & Qua Astigmatism & 0.1 & 0.05 & 0.06 & 0.06 & 0.02 \\
\hline 20 & 9 & 1 & Qua Coma & 0.1 & 0.05 & 0.06 & 0.06 & 0.02 \\
\hline 21 & 10 & 0 & Qua Spherical & 0.1 & 0.05 & 0.06 & 0.06 & 0.02 \\
\hline 22 & 12 & 0 & Qin Spherical & 0.1 & 0.05 & 0.06 & 0.06 & 0.02 \\
\hline
\end{tabular}

Note: as will be discovered in Section 5, the Baseline Telescope design meets the LOS stability allocation by several orders of magnitude. And, while the telescope also meets the Inertial and Thermal stability specification, it meets them with less margin. Therefore, we plan to reallocate the Figure 4 error budget. 


\section{OPTICAL DESIGN TOLERANCE SENSITIVITY}

To achieve the Wavefront (WFE) Stability and Line of Sight (LOS) Stability specifications requires an ultra-stable optomechanical telescope structure that can align the primary, secondary and tertiary mirrors to each other and maintain that alignment. Rigid body motions of the primary, secondary and tertiary mirrors introduce WFE and LOS errors. As discussed in Stahl $2017^{5}$, Zemax tolerance analysis of the baseline optical design provides the LOS and WFE sensitivity to rigid body motions of the primary, secondary and tertiary mirror alignment for the baseline F/2.5 optical design.

From these sensitivities, an LOS error budget was allocated for each rigid body degree of freedom (DOF) (Figure 5). Given, as discussed in Section 5, that the two rigid body motion response modes that have the highest impact on LOS stability are the Primary Mirror X-and Y-decenter modes, as much tolerance as possible is allocated to these modes.

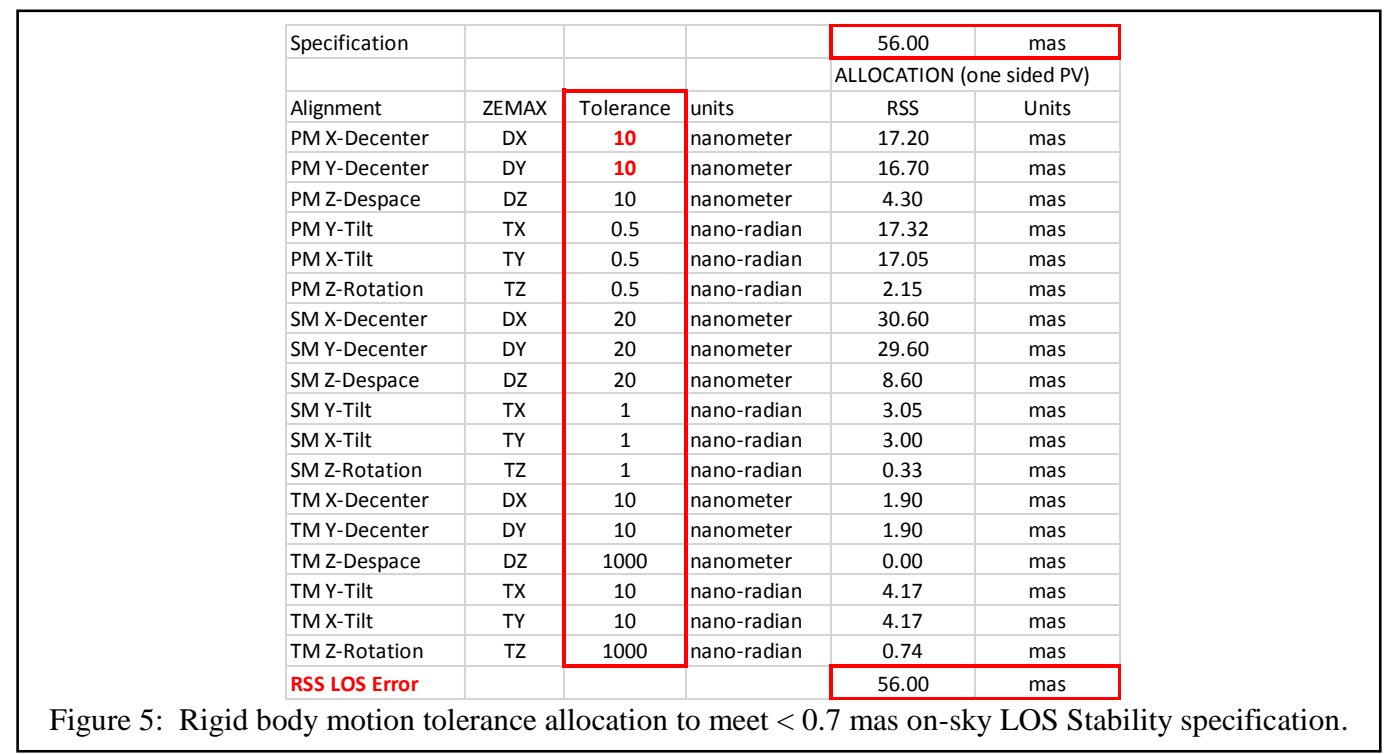

Then, using the Zemax analysis, the Figure 5 LOS allocation were checked for consistency with the Figure 4 WFE error budget allocation (Figure 6). One might be concerned by the low margin, but as shown in Section 5, predicted performance is several orders of magnitude better than this requirement.

\begin{tabular}{|c|c|c|c|c|c|c|c|}
\hline \multirow[t]{2}{*}{ RSS Allocation } & \multirow[t]{2}{*}{$100 \%$} & \multirow[t]{2}{*}{$50 \%$} & \multirow[t]{2}{*}{$60 \%$} & \multirow[t]{2}{*}{$60 \%$} & \multirow[t]{2}{*}{$20 \%$} & \multicolumn{2}{|c|}{ Zerodur Mirror (16 Feb 18) on 3pt mount } \\
\hline & & & & & & & 0.7 mas LOS \\
\hline & VVC-6 Allowable & LOS & Inertial & Thermal & Reserve & & RSS Wavefront \\
\hline Aberration & [pm rms] & [pm rms] & [pm rms] & [pm rms] & [pm rms] & MARGIN & (pm rms) \\
\hline TOTAL RMS & 416 & 208 & 250 & 250 & 83 & 1.4 & 151 \\
\hline Tilt & 0 & & & & & & 21.37 \\
\hline Power (Defocus) & 250 & 125 & 150 & 150 & 50 & 0.9 & 145.20 \\
\hline Pri Astigmatism & 200 & 100 & 120 & 120 & 40 & 3.1 & 32.51 \\
\hline Pri Coma & 175 & 87.5 & 105 & 105 & 35 & 11.6 & 7.51 \\
\hline Pri Spherical & 200 & 100 & 120 & 120 & 40 & 264.9 & 0.38 \\
\hline Pri Trefoil & 2.6 & 1.3 & 1.56 & 1.56 & 0.52 & 3.7 & 0.35 \\
\hline Sec Astigmatism & 0.35 & 0.175 & 0.21 & 0.21 & 0.07 & 1.3 & 0.14 \\
\hline Sec Coma & 0.35 & 0.175 & 0.21 & 0.21 & 0.07 & 7.9 & 0.02 \\
\hline Sec Spherical & 0.35 & 0.175 & 0.21 & 0.21 & 0.07 & 176.2 & 0.00 \\
\hline Pri Tetrafoil & 0.35 & 0.175 & 0.21 & 0.21 & 0.07 & 43.3 & 0.00 \\
\hline Sec Trefoil & 0.35 & 0.175 & 0.21 & 0.21 & 0.07 & 95.2 & 0.00 \\
\hline Ter Astigmatism & 0.1 & 0.05 & 0.06 & 0.06 & 0.02 & 103.7 & 0.00 \\
\hline Ter Coma & 0.1 & 0.05 & 0.06 & 0.06 & 0.02 & 843.0 & 0.00 \\
\hline Ter Spherical & 0.1 & 0.05 & 0.06 & 0.06 & 0.02 & 4157.4 & 0.00 \\
\hline Pri Pentafoil & 0.35 & 0.175 & 0.21 & 0.21 & 0.07 & & \\
\hline Sec Tetrafoil & 0.1 & 0.05 & 0.06 & 0.06 & 0.02 & & \\
\hline Ter Trefoil & 0.1 & 0.05 & 0.06 & 0.06 & 0.02 & & \\
\hline Qua Astigmatism & 0.1 & 0.05 & 0.06 & 0.06 & 0.02 & & \\
\hline Qua Coma & 0.1 & 0.05 & 0.06 & 0.06 & 0.02 & & \\
\hline Qua Spherical & 0.1 & 0.05 & 0.06 & 0.06 & 0.02 & & \\
\hline Qin Spherical & 0.1 & 0.05 & 0.06 & 0.06 & 0.02 & & \\
\hline
\end{tabular}




\section{OPTICAL TELESCOPE CONCEPT}

The current 'baseline' HabEx optical telescope design (Figure 7) is a modification of the initial concept published in Stahl $2017^{5}$ - which was itself based on the HabEx-4 design concept study performed by the NASA Marshall Space Flight Center (MSFC) Advanced Concept Office (ACO) in November 2015 and published in August 2016. ${ }^{\mathbf{1 3}}$ CAD and FEM models have been developed for the Baseline Design and are being used for STOP analysis. The optical telescope assembly (OTA) consists of the primary mirror assembly, secondary mirror assembly, secondary mirror tower, stray-light tube, forward scarf, and science instrument bays. The forward scarf angle (currently set at 45 degrees) determine the closest angle of observation to the sun. The baffle tube and SM tower are the optical bench which connects and retains optical alignment between the PMA and SMA. The stray-light baffles double as stiffening rings. Because the OTA is off axis, the rings are not continuous. Thus, external gussets span the structural gap from tube to tower. The OTA is physically separate from the spacecraft which includes the solar array sunshield. The size of the solar arrays on the bottom are driven by thermal power requirements during anti-sun pointing. Instead of reaction wheels, cold gas thrusters are used for slewing the observatory and micro-thrusters are used for fine pointing control during science observations. The OTA and spacecraft connect only at the interface ring. This ring is also the interface between the payload and the Space Launch System (SLS).

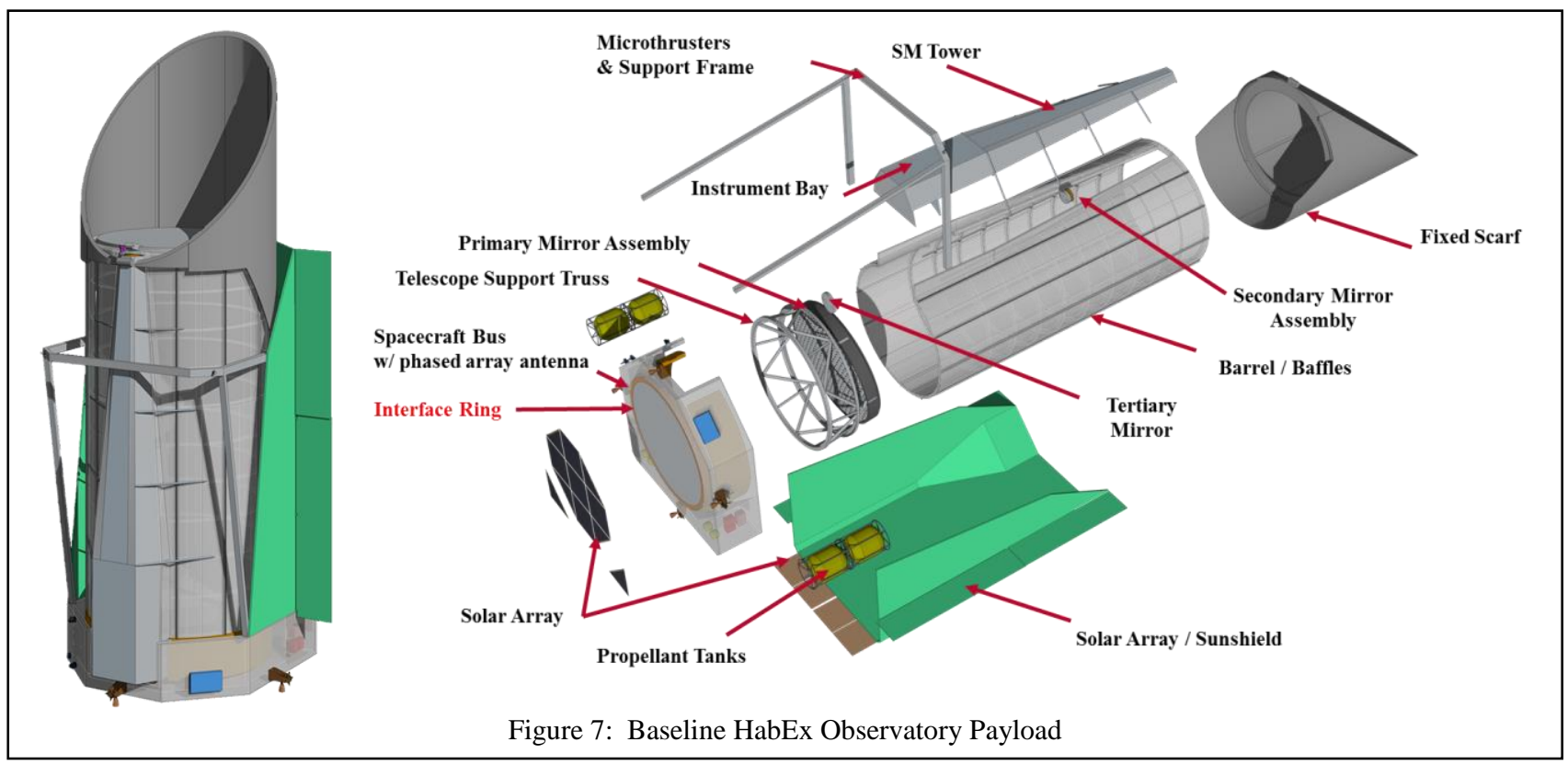

The Baseline HabEx Observatory is designed specifically for the SLS Block IB mass and volume capacities, and launch environment. ${ }^{13}$ The payload fits inside the SLS 8.4 meter fairing (Figure 8) without the need for any deployments. The projected total mass of $15 \mathrm{mt}$ mass (Table 3 ) has nearly $200 \%$ margin against the SLS Block-2 mass capacity of $44 \mathrm{mt}$ to SE-L2. And, its structure is sized for a $3.5 \mathrm{~g}$ axial and $1.5 \mathrm{~g}$ lateral launch load. A ground rule for the study was that every proposed system, subsystem or component of the spacecraft (including: propulsion; attitude control; power; avionics; communication; command and data handling; etc.) should be at TRL-9 except for the primary mirror assembly, active thermal control system, and science instruments. HabEx-4 was designed for a 15 year operational life at SE-L2 with no servicing. Its propellant load is sized with a $25 \%$ reserve against this 15 year operational life requirement.

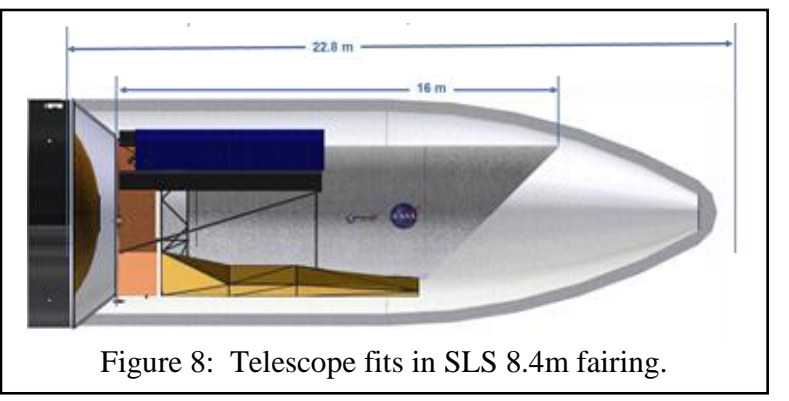

\begin{tabular}{|l|c|c|c|}
\hline \multicolumn{4}{|c|}{ Table 3: HabEx Observatory Payload Mass Estimate } \\
\hline Element & CBE $[\mathrm{kg}]$ & 30\% Reserve $[\mathrm{kg}]$ & Total [kg] \\
\hline Telescope & 4310 & 1293 & 5603 \\
\hline Science Instruments & 1500 & 450 & 1950 \\
\hline Spacecraft & 4500 & 1350 & 5850 \\
\hline Interface Ring & 210 & 63 & 273 \\
\hline PAF & TBD & & \\
\hline \hline Payload Dry Mass & 10520 & & 13676 \\
\hline \hline Propellant & 1700 & & 1700 \\
\hline \hline Payload Wet Mass & 21220 & & 15276 \\
\hline \hline
\end{tabular}


The largest mass element of the baseline OTA is the primary mirror (PM) and its support system (Table 4). The baseline primary mirror is a 4-m open-back iso-grid Zerodur ${ }^{\odot}$ mirror. This mirror was selected because Schott has demonstrated a routine ability to fabricate 4.2-m diameter Zerodur substrates and turn them into lightweight mirrors via their extreme-lightweight Zerodur Mirror (ELZM) machining process. The baseline mirror design, selected after an extensive trade study ${ }^{14}$, provides an excellent balance between mass and stiffness. The mirror's free-free first mode frequency is $78 \mathrm{~Hz}$. Its mounted first mode frequency is $58 \mathrm{~Hz}$. And, when integrated into the telescope, its first

\begin{tabular}{|l|c|}
\hline \multicolumn{2}{|l|}{ Table 4: HabEx Optical Telescope Mass Estimate } \\
\hline Component & CBE $[\mathrm{kg}]$ \\
\hline Primary Mirror & 1356 \\
\hline Primary Mirror Support & 1001 \\
\hline Secondary Mirror Assembly & 11 \\
\hline Secondary Mirror Tower & 376 \\
\hline Stray-light Baffle Tube & 1536 \\
\hline Tertiary Mirror Assembly & 20 \\
\hline \hline Total OTA Mass & 4310 \\
\hline \hline
\end{tabular}
mode frequency is $38 \mathrm{~Hz}$. An alternative $\mathrm{ULE}^{\odot}$ mirror has also been designed. It has a mass of $924 \mathrm{~kg}$ and a free-free first mode frequency of $128 \mathrm{~Hz}$. There are two reasons to consider this mirror. First, it has lower mass. But more importantly, it has higher stiffness - which means that it will have smaller dynamic inertial wavefront error. Note, as discussed in Section 2.1, all of the PM modal frequencies are above the control frequency of the anticipated LOWFS. Finally, regarding mass, it has one very important advantage. It provides thermal capacity and enable a thermally stable primary mirror. Another reason that Zerodur was selected is its excellent CTE homogeneity. The Primary Mirror Support system is sized to accommodate a launch constraint system. The composite material for the tube and truss structure is M46J with quasiisotopic laminate properties of $25 \% 0-\mathrm{deg}, 50 \% 45-\mathrm{deg}, 25 \% 90-\mathrm{deg}$ and a density of $1.58 \mathrm{~g} / \mathrm{cm}^{3}$.

\section{TELESCOPE STRUCTURAL THERMAL OPTO-MECHANICAL PERFORMANCE}

The fundamental question is: Does the baseline design achieve the specified LOS and WFE stability? To answer this question, FEM and thermal models of the baseline telescope integrated with the spacecraft were constructed. These models were exercised to determine whether or not the baseline design meets the stability error budget defined in Figure 4. Specifically, they were used to analyze LOS Jitter, Inertial WFE Stability and Thermal Stability.

\subsection{Predicted Line of Sight (LOS) Stability Performance}

To determine LOS jitter performance, a FEM of the baseline telescope and spacecraft structure was constructed and exposed to a mechanical disturbance spectrum. Critical Damping was set at $0.05 \%$. The NASTRAN Multi Point Constraint (MPC) function was used to determine rigid body displacements of the primary and secondary mirrors relative to the fold mirror (Figure 9). Another MPC determined rigid body displacements between the PM and SM. These displacements were quantified for mechanical disturbance noise spectrum from 0 to $500 \mathrm{~Hz}$.

In the Stahl 2017 paper $^{5}$, it was shown that the initial telescope design, if exposed to the JWST reaction wheel noise specification with JWST passive vibration isolation, did not meet the LOS jitter specification. But, it might meet the specification with $60 \mathrm{~dB}$ of active vibration isolation. And, it would meet the specification if the

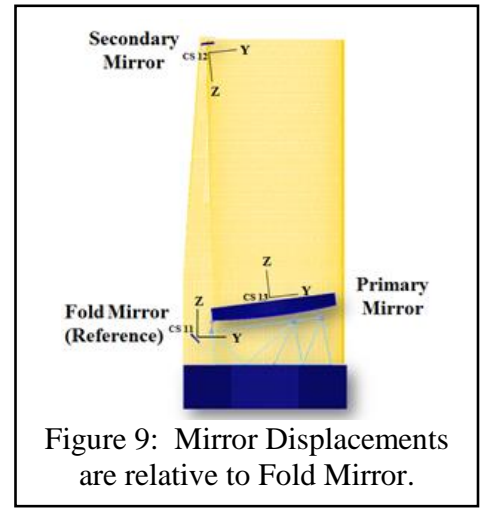
reaction wheels were replaced by micro-thrusters.

To improve LOS jitter performance for the baseline design, the primary support system was redesigned to increase its first mode frequency from 25 to $38 \mathrm{~Hz}$. And, micro-thrusters were selected instead of reaction wheels. To minimize mechanical disturbance, cold gas thrusters are used to slew and point the telescope, then turned off. Micro-thrusters, attached to the spacecraft and isolated from the telescope, are used to maintain pointing for the duration of a science exposure. Micro-thrusters run continuously with variable thrust proportional to applied current, Figure 10 shows a measured noise PSD for a colloidal micro-thruster. Because the data is noisy and has not been measured beyond $5 \mathrm{~Hz}$, the MPC LOS analysis assumes that each micro-thruster has a flat 0.1 micro-Newton noise spectrum from 0 to $500 \mathrm{~Hz}$. Please note that the baseline design has multiple micro-thruster 'heads' and that each head consists of four individual thrusters.

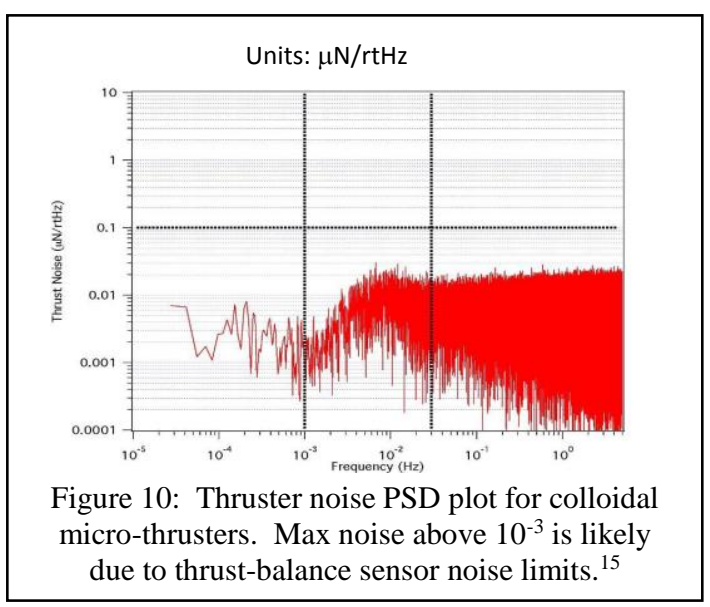


Figure 11 shows the amplitudes versus frequency for each rigid body degree of freedom between the Primary and Secondary Mirrors produced by the micro-thruster specification of 0.1 micro-Newtons. Note that the amplitudes were multiplied by a $2 X$ Model Uncertainty Factor (MUF) for frequencies below $20 \mathrm{~Hz}$ and a 4X MUF for frequencies above $20 \mathrm{~Hz}$. The red lines are the tolerances summarized in Table 2. Obviously, the predicted LOS performance of the baseline design is better than the requirement by at least 100X. As shown in Figure 6, if the telescope can achieve its LOS jitter specification, it will also achieve its LOS WFE stability allocation. And, given the amount of LOS WFE stability margin, the error budget should be reallocated to provide a larger tolerance for inertial and thermal WFE.

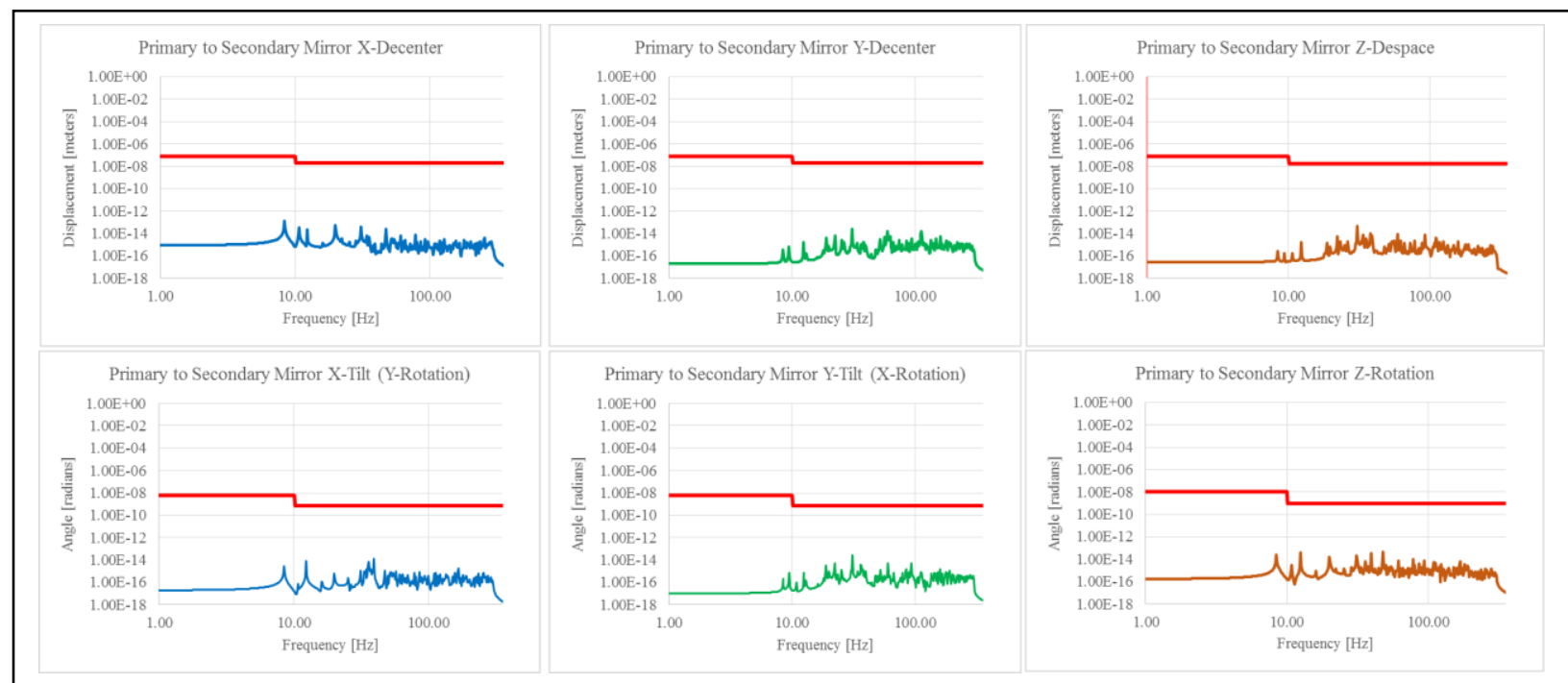

Figure 11: Primary to Secondary Mirror Rigid Body Amplitudes for 0.1 micro-Newton 'white' noise spectrum

\subsection{Predicted Inertial WFE Stability Performance}

Inertial WFE is not the same as modal motion. Inertial WFE occurs when the primary mirror (secondary, etc.) is accelerated by a mechanical motion (such as vibration or modal motion). This acceleration causes the mirror to react against its support structure physically bending the mirror.

For this paper, we have developed a simple first-order method to estimate inertial wavefront stability based on the equivalence of gravity and acceleration; and, assuming linearity of material properties (such as Young's Modulus) for small accelerations.

To estimate inertial WFE produced by the 0.1 micro-N micro-thruster specification, we start by calculating the primary mirror's mounted $1 \mathrm{G}$ gravity sag for the orthogonal XYZ directions (Figure 12). Then, we decompose each into Zernike polynomials and scale them from $1 \mathrm{G}$ to 0.1 micro-N. Finally, the inertial WFE estimate is obtained by RSS'ing the XYZ Zernike coefficients. Figure 13 shows that, to first order, the baseline 4-m 78-Hz free-free Zerodur mirror's estimated inertial WFE meets the allocation defined in Figure 4 . The most challenging allocation is primary tetrafoil with a 6.8 margin. Design refinements may reduce the PM's contribution to this error.

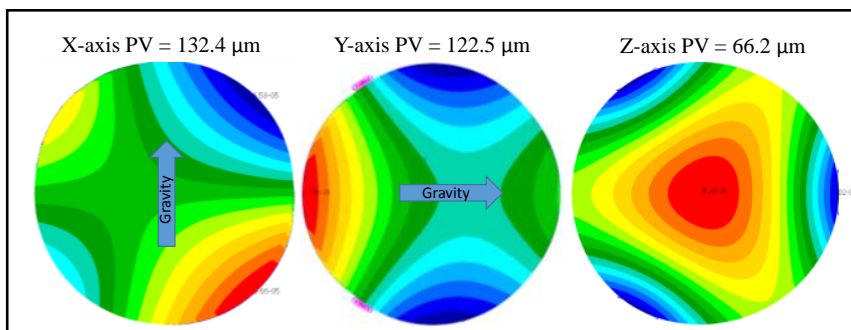

Figure 12: XYZ gravity sag of the baseline Zerodur mirror

\begin{tabular}{|c|c|c|c|c|c|c|}
\hline \multirow[b]{4}{*}{ Aberration } & \multirow[b]{4}{*}{ Tolerance [pm rms] } & \multirow[b]{4}{*}{ MARGIN } & \multirow{4}{*}{$\begin{array}{c}\text { OUTPUT } \\
0.1 \mu \mathrm{N} \\
\text { RSS Wavefront } \\
\text { (pm rms) } \\
\end{array}$} & \multirow{2}{*}{\multicolumn{3}{|c|}{$\begin{array}{l}\text { INPUT } \\
\text { PV Zernike } 1 \mathrm{G} \text { Deformation }\end{array}$}} \\
\hline & & & & & & \\
\hline & & & & \multirow{2}{*}{$\begin{array}{l}\text { X-Axis } \\
\text { (waves) }\end{array}$} & \multirow{2}{*}{$\begin{array}{l}\text { Y-Axis } \\
\text { (waves) }\end{array}$} & \multirow{2}{*}{$\begin{array}{l}\text { Z-Axis } \\
\text { (waves) }\end{array}$} \\
\hline & & & & & & \\
\hline Bias & & & 0.003 & 0.00682 & 0.00385 & 0.24025 \\
\hline Tilt & & & 0.005 & 0.26243 & 0.25445 & 0.73308 \\
\hline Power (Defocus) & 150 & 613.5 & 0.245 & -0.39796 & -0.22801 & 32.78968 \\
\hline Pri Astigmatism & 120 & 217.4 & 0.552 & 73.99304 & 74.07263 & 1.8843 \\
\hline Pri Coma & 105 & 17209.0 & 0.006 & 0.55113 & 0.86725 & 0.85432 \\
\hline Pri Spherical & 120 & 4444.5 & 0.027 & 0.02169 & 0.01248 & -4.67483 \\
\hline Pri Trefoil & 1.56 & 9.6 & 0.163 & 0.93822 & 0.78428 & 35.57098 \\
\hline Sec Astigmatism & 0.21 & 13.1 & 0.016 & 2.77 & 2.75838 & 0.46075 \\
\hline Sec Coma & 0.21 & 440.2 & 0.000 & 0.0407 & 0.0704 & 0.0988 \\
\hline Sec Spherical & 0.21 & 151.6 & 0.001 & 0.00723 & 0.00436 & 0.28358 \\
\hline Pri Tetrafoil & 0.21 & 6.8 & 0.031 & 5.29101 & 5.42104 & 0.47626 \\
\hline Sec Trefoil & 0.21 & 18.2 & 0.012 & 0.11876 & 0.09066 & 3.08851 \\
\hline Ter Astigmatism & 0.06 & 74.8 & 0.001 & 0.1648 & 0.15934 & 0.03734 \\
\hline Ter Coma & 0.06 & 472.1 & 0.000 & 0.02756 & 0.02649 & 0.00939 \\
\hline Ter Spherical & 0.06 & 231.6 & 0.000 & 0.00148 & 0.00122 & -0.06016 \\
\hline Pri Pentafoil & 0.21 & 12.5 & 0.017 & 3.2123 & 3.14561 & 0.04258 \\
\hline Sec Tetrafoil & 0.06 & 16.2 & 0.004 & 0.73808 & 0.77607 & 0.02492 \\
\hline Ter Trefoil & 0.06 & 347.8 & 0.000 & 0.01926 & 0.01775 & 0.04657 \\
\hline Qua Astigmatism & 0.06 & 3167.4 & 0.000 & 0.00291 & 0.00446 & 0.00322 \\
\hline Qua Coma & 0.06 & 622.2 & 0.000 & 0.02295 & 0.02326 & 0.00689 \\
\hline Qua Spherical & 0.06 & 2300.1 & 0.000 & -0.00128 & -0.00028 & 0.00657 \\
\hline Qin Spherical & 0.06 & 1085.8 & 0.000 & -0.00177 & -0.00043 & 0.01532 \\
\hline
\end{tabular}

Figure 13: Baseline Zerodur Mirror meets with margin inertial WFE allocation for 0.1 micro-Newton XYZ 'white' noise. 


\subsection{Predicted Thermal WFE Stability Performance}

Thermal errors occur when a telescope is slewed relative to the Sun. Thermal load changes cause the structure holding the mirrors to expand/contract (resulting in alignment drift) and they cause the mirrors themselves to change shape - due to coefficient of thermal expansion (CTE). Mirrors change shape when their bulk temperature or thermal gradient changes.

Thermal Desktop was used to estimate thermal WFE stability of the baseline telescope integrated with the spacecraft (including sun-shield and MLI) for a worst case observing scenario - the telescope points at a guide start with which to 'dig' the dark-hole, then slews to the target star and observes for 360 minutes. As shown in Figure 14, after 90 min the total WFE changes by 1 pico-meters rms; and, after $360 \mathrm{~min}$ it changes by $8 \mathrm{pm}$ rms. Figure 15 shows this thermal WFE decomposed into Zernike polynomials. The largest error is power, but the 360 minute amplitude of 8 picometers rms is well below the $150 \mathrm{pm}$ rms allocation for this term. The most important term is trefoil. Its $0.5 \mathrm{pm}$ rms allocation is achieved in approximately 90 minutes. Please note that for this analysis, the CTE of the primary mirror is assumed to be perfectly homogeneous. The slow rate of thermal WFE change is mostly because of the primary mirror's thermal mass. ${ }^{16}$

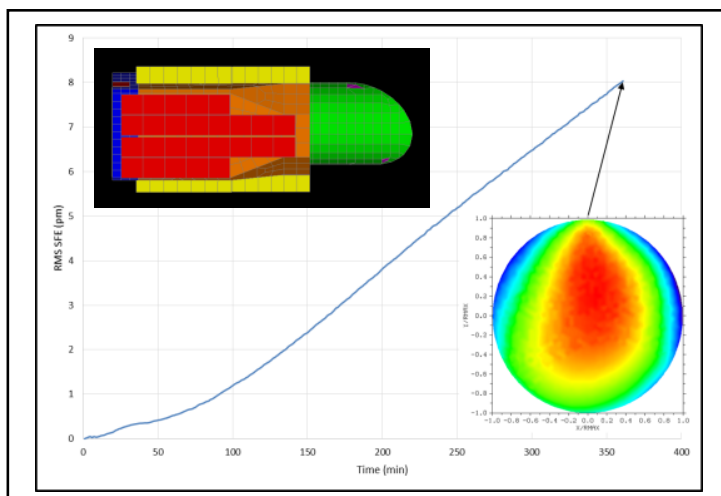

Figure 14: RMS WFE produced by a 20 degree slew.

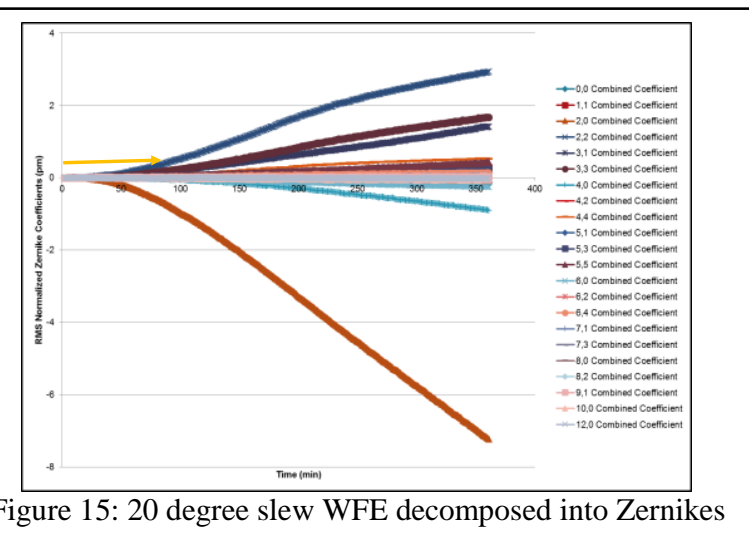

Figure 15: 20 degree slew WFE decomposed into Zernikes

Another important source of thermal WFE is CTE inhomogeneity. CTE inhomogeneity causes mirrors to change shape as a function of bulk temperature or thermal gradient change. Typically, to mitigate this issue, space telescopes use low coefficient of thermal expansion materials such as Zerodur ${ }^{\odot}$ ceramic or Ultra-Low Expansion $\left(\mathrm{ULE}^{\odot}\right.$ ) glass. The HabEx baseline primary mirror is Zerodur. One method to estimate thermal WFE with CTE inhomogeneity is the measure the thermal WFE deformation of a representative mirror and assume that CTE is linear with temperature. As part of the Advanced Mirror Technology Development (AMTD) project, a 1.2-m ELZM was measured to have an $11.3 \mathrm{~nm}$ rms WFE over a $62 \mathrm{~K}$ thermal range. ${ }^{17}$ Figure 16 shows that for this mirror to meet the Figure 4 error budget allocation requires that the mirror's temperature be stable to approximately $1 \mathrm{mK}$.

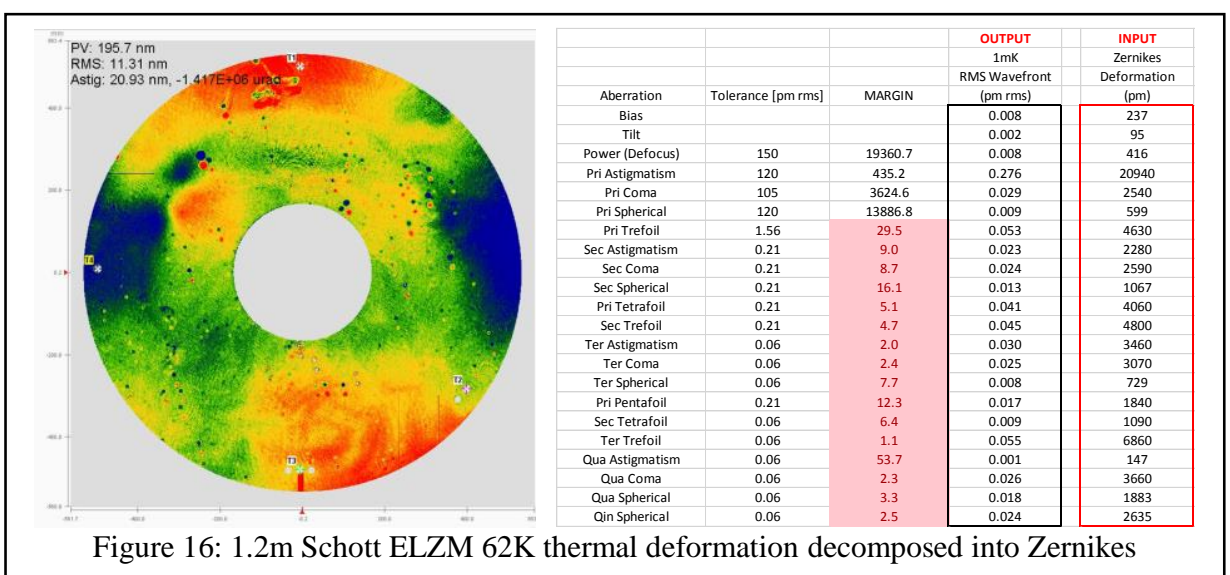

Please note that it is not necessary to keep the primary mirror's thermal environment stable to $1 \mathrm{mK}$. Because of its thermal mass, the primary mirror responds slowly to changes in its thermal environment. HabEx is baselining a predictive thermal control system to monitor both the thermal load (as a function of sun angle) and mirror's temperatures, and modify the thermal environment to keep the mirror at a constant temperature. ${ }^{12}$ 


\section{CONCLUSION}

The Habitable Exoplanet Observatory Mission (HabEx) is under study for the 2020 Astrophysics Decadal Survey. Its goal is to image and spectroscopically characterize planetary systems in around nearby sun-like stars. Critical to achieving the HabEx science goals is a large, ultra-stable UV/Optical/Near-IR (UVOIR) telescope. The desired telescope is a 4-meter off-axis unobscured three-mirror-anastigmatic, diffraction limited at $400 \mathrm{~nm}$ with wavefront stability on the order of a few 10s of picometers. The baseline HabEx telescope is designed using standard engineering practice and its design 'closes'. The telescope's predicted Structural Thermal Optical Performance meets with margin its specified performance error budget allocations for Line of Sight Jitter, LOS Wavefront Error, Inertial WFE and Thermal WFE. Key to meeting its LOS and Inertial specifications is the choice to use micro-thrusters for pointing control instead of reaction wheels. The baseline observatory design fits with margin within the mass and volume constraints of the SLS Block-2 8.4-m fairing.

\section{ACKNOWLEDGEMENTS}

This paper is the work of the NASA MSFC HabEx Team and our JPL Collaborators. MSFC Team: Michael Effinger, Scott Smith, Thomas Brooks, Jacqueline Davis, Brent Knight, Mark Stahl; Willian Arnold (AI Solution); Mike Baysinger, Jay Garcia, Ron Hunt, Andrew Singleton, Mary Caldwell and Melissa Therrell (ESSA); Bijan Nemati (UAH); and interns Jonathan Gaskin (UNCC), Jonathan McCready (NCSU), and Hao Tang (UoMI). JPL Team: Keith Warfield, Gary Kuan, Stefan Martin, Velibor Cormarkovic, Scott Howe, Juan Villalvazo, Stuart Shaklan, and Team X.

\section{REFERENCES}

[1] Committee for a Decadal Survey of Astronomy and Astrophysics; National Research Council, New Worlds, New Horizons in Astronomy and Astrophysics, The National Academies Press, Washington, D.C., 2010.

[2] NASA Space Technology Roadmaps and Priorities: Restoring NASA's Technological Edge and Paving the Way for a New Era in Space, NRC Report, 2012.

[3] Hertz, Paul, "Planning for the 2020 Decadal Survey: An Astrophysics Division White Paper", January 4, 2015, available at science.nasa.gov/astrophysics/documents/.

[4] NASA Town Hall, AAS Winter Meeting, Kissimmee, FL, 2016.

[5] Stahl, H. Philip, "Overview of a telescope concept design for the Habitable-zone Exoplanet Direct Imaging Mission", Proc. SPIE 10398, UV/Optical/IR Space Telescopes and Instruments: Innovative Technologies and Concepts VIII, 1039806 (5 September 2017); doi: 10.1117/12.2275192; http://dx.doi.org/10.1117/12.2275192

[6] Krist, Trauger, Unwin and Traub, "End-to-end coronagraphic modeling including a low-order wavefront sensor", SPIE Vol. 8422, 844253, 2012; doi: 10.1117/12.927143

[7] Harvey, James E. and Christ Ftaclas, "Diffraction effects of telescopes secondary mirror spiders on various imagequality criteria", Applied Optics, Vol. 34, No. 28, pp-6337, 1 Oct 1995.

[8] Morgan, Rhonda H., et. al., "HabEx yield modeling with for systems engineering", SPIE 10398-3, 2017.

[9] NASA, Exo-C: Imaging Nearby Worlds, CL\#15-1197, March 2015 https://exep.jpl.nasa.gov/stdt/Exo-C_Final_Report_for_Unlimited_Release_150323.pdf

[10] Stuart Shaklan, private communication, 10 Aug 2017

[11] Garrett Ruane, private communication, June 2017

[12] Brooks, Thomas E., "Predictive thermal control applied to HabEx", Proc. SPIE 10398, UV/Optical/IR Space Telescopes and Instruments: Innovative Technologies and Concepts VIII, 1039814 (5 September 2017); doi: $10.1117 / 12.2274338$

[13] Stahl, H. Philip, et. al., "Designing astrophysics missions for NASA's Space Launch System," J. Astron. Telesc. Instrum. Syst. 2(4), 041213 (2016), doi: 10.1117/1.JATIS.2.4.041213.

[14] Arnold, William R., H. Philip Stahl, "Design trade Study for a 4-meter off-axis primary mirror substrate and mount for the Habitable-zone Exoplanet Direct Imaging Mission", Proc. SPIE 10398, UV/Optical/IR Space Telescopes and Instruments: Innovative Technologies and Concepts VIII, 1039808 (5 September 2017); doi: 10.1117/12.2275193;

[15]Ziemer, et. al., "Colloid Micro-Newton Thrusters for Precision Attitude Control”, CL\#17-2067, April 2017.

[16] Brooks, Thomas, H. P. Stahl, William R. Arnold, "Advanced Mirror Technology Development (AMTD) thermal trade studies", Proc. SPIE. 9577, (September 23, 2015) doi: 10.1117/12.2188371

[17]Brooks, Thomas E., Ron Eng, Tony Hull, H. Philip Stahl, "Modeling the Extremely Lightweight Zerodur Mirror (ELZM) thermal soak test", Proc. SPIE 10374, Optical Modeling and Performance Predictions IX, 103740E (6 September 2017); doi: 10.1117/12.2274084 